\title{
Primary Retroperitoneal Teratoma with Predominant Neurogenic Elements Masquerading as Adrenal Tumor
}

\author{
Sonam SHARMA, Leelavathi DAWSON, Ashish Kumar MANDAL \\ Department of Pathology, Vardhman Mahavir Medical College and Safdarjung Hospital, NEW DELHI, INDIA
}

\begin{abstract}
Primary retroperitoneal teratomas are uncommon extragonadal nonseminomatous germ cell tumors that are composed of well differentiated parenchymal tissues which are derived from more than one of the three embryonic germ cell layers. Here we report an unusual and first of its kind, a case of primary mature cystic retroperitoneal teratoma mimicking as adrenal tumor in a 7-month-old female in which the tumor was predominantly composed of neurogenic tissue histologically which is unlike the usual pattern seen in the teratomas.
\end{abstract}

Key Words: Retroperitoneal teratoma, Neurogenic elements, Adrenal neoplasm

\section{INTRODUCTION}

Teratomas also known as dysembryoma, teratoblastoma, organoid tumor and teratoid tumor, are encapsulated neoplasms, that are the most common form of all germ cell tumors (GCTs) and belong to nonseminomatous group of GCTs (1). They arise from abnormal development of pluripotent cells: germ cells and embryonal cells, which in turn greatly influences the age of presentation and involved location. Teratomas of germ cell origin can be congenital or acquired and are usually gonadal. In contrast, teratomas of embryonic cell sources, which are always congenital and are usually found in extragonadal (15\%) locations, such as sacrococcygeal, intracranial, cervical, mediastinal and retroperitoneal (2). Major differences in their clinical behavior suggest that gonadal and extragonadal tumors are biologically different, though histological, serological, and cytogenetic characteristics of all GCTs are similar (3). The present case study describes a child with an atypical presentation of a rare case of primary retroperitoneal teratoma which posed a diagnostic challenge and is first of its kind in terms of histology to be reported in the world literature.

\section{CASE REPORT}

A 7-month-old female presented with a gradually increasing lump in the left upper abdomen, which was first noticed 3 months back. There was no history of fever, weight loss, gastrointestinal, genito-urinary or respiratory disturbances. Developmental milestones of the child were

(Turk Patoloji Derg 2019, 35:69-73)

Received : 26.03.2016 Accepted : 10.06.2016 within normal range, with normal birth history. Her past history and medical history were non-contributory.

On physical examination, a large intra-abdominal mass was palpable in the left upper quadrant which was also extending into the left epigastric and left lumbar region. It measured around $9 \times 8 \mathrm{~cm}$ and was firm, non-tender, moving with respiration and dull on percussion. The overlying skin was unremarkable. All other systemic examinations were within normal limits. Routine haematological investigations were unremarkable. Urine and blood cultures were negative. Kidney, liver function tests, and X-ray of chest were normal. Serum antibodies to human immunodeficiency virus and hepatitis B surface antigen were negative.

Abdominal ultrasonography (USG) showed a large, multicystic mass located between the spleen and left kidney. There was no evidence of calcification in the tumor mass or ascitis. Contrast enhancement computed tomography (CECT) scan of abdomen and pelvis demonstrated a large well circumscribed predominantly cystic retroperitoneal mass occupying predominantly the left suprarenal region (Figure 1A,B). It measured about $9.9 \times 8.8 \times 6.8 \mathrm{~cm}$ and it showed multiple septae, a tiny fat and an enhancing soft tissue attenuation area. No calcification was seen. The mass displaced the aorta, celiac axis and superior mesenteric vessels to the contralateral side and the left kidney caudally with indentations to its contour. The left renal vein was displaced antero-medially and draped along the medial margin of the mass. The left adrenal gland could not be detected separately from the mass. The body and

Correspondence: Sonam SHARMA

Vardhman Mahavir Medical College and Safdarjung Hospital,

Department of Pathology, NEW DELHI, INDIA

E-mail: drsonamsharma@gmail.com Phone: +9999841393 

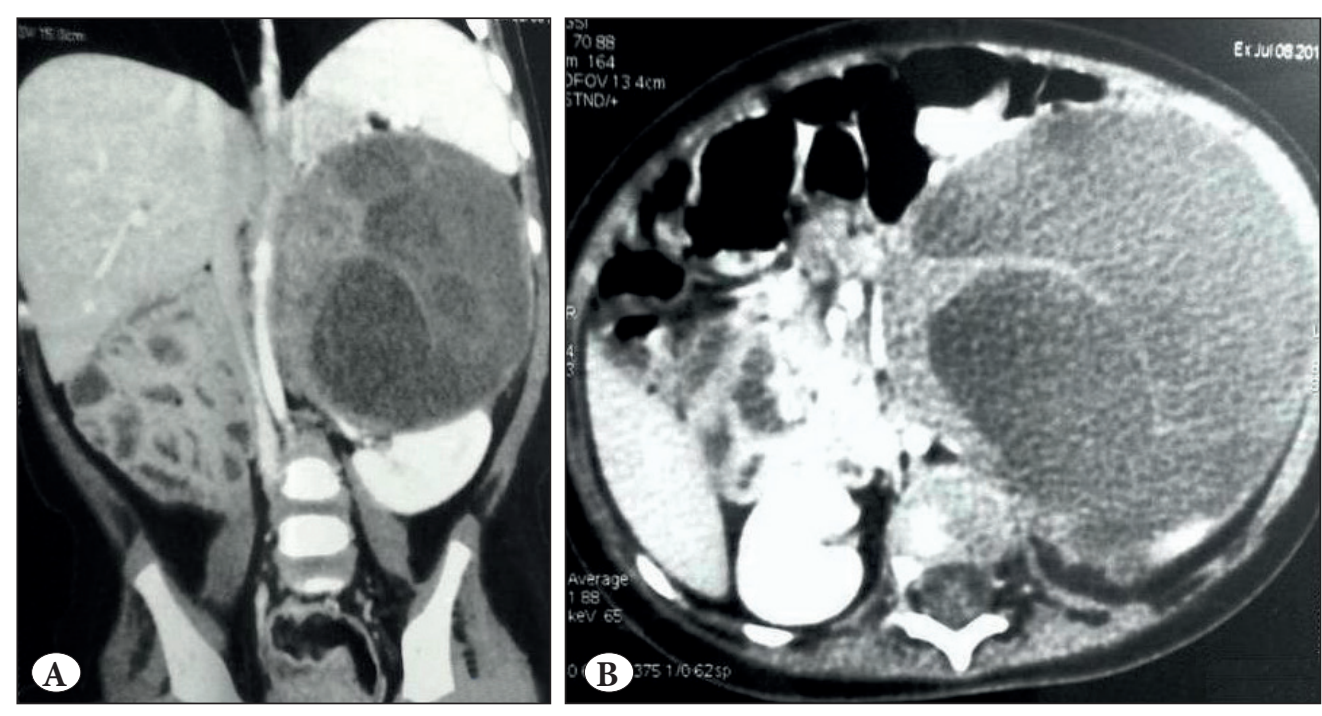

Figure 1: A,B) CECT abdomen revealing a multicystic mass in the retroperitoneum.

tail of pancreas were displaced anteriorly while descending colon and small bowel loops were displaced to right side. There was no evidence of any significant abdominal and pelvic lymphadenopathy or distant metastasis. Rest of the abdominal and pelvic organs were unremarkable. Based on these findings, a radiological suspicion of a cystic change in a solid tumour originating from left adrenal gland (as the normal left adrenal gland could not be recognized) or a retroperitoneal cystic teratoma was made.

Laboratory investigations indicating a functioning adrenal tumour, consisting of plasma and urinary levels of catecholamines, rennin, aldosterone, cortisol, adrenocorticotrophic hormone levels were within normal limits. Tumor markers such as serum alpha-fetoprotein (AFP), lactate dehydrogenase (LDH), neuron-specific enolase (NSE), carcino embryonic antigen (CEA) and carbohydrate antigen 19-9 (CA 19-9), were also estimated. Serum values of AFP $(18.9 \mu \mathrm{g} / \mathrm{dL})$, CEA $(6.6 \mathrm{ng} / \mathrm{ml})$, CA19-9 $(50.2 \mathrm{U} / \mathrm{ml})$ were slightly higher whereas LDH and NSE were within normal range, further ruling out the possibility of left adrenal gland being the origin of this mass.

On exploratory laparotomy, a large well defined cystic retroperitoneal mass occupying the left suprarenal area, between the spleen and left kidney was seen. The left adrenal gland was compressed and adhered to the tumor mass. The mass abutted the left kidney and displaced it inferiorly. The renal vessels were stretched and adherent to the mass.The transverse and left colon were compressed and displaced anteriorly whereas tail and body of the pancreas were displaced posteriorly. No invasion into the aorta or inferior vena cava was seen. No palpable regional nodes were identified. The tumor mass was completely excised and sent for histopathological examination.
Gross examination of the specimen received showed a well-circumscribed cystic mass measuring $9.8 \times 9 \times$ $8 \mathrm{~cm}$ with an intact and smooth surface. On incision, brownish fluid admixed with mucoid/jelly like material came out and thin walled cyst was left. On cut section, multiloculated cysts were seen along with a very small solid grey yellow area measuring $0.8 \times 0.8 \times 0.5 \mathrm{~cm}$ (Figure $2 \mathrm{~A}, \mathrm{~B}$ ). Histopathological examination of cyst wall and small solid area showed predominantly mature neural tissue (Figure 3A). A glandular structure lined by ciliated columnar epithelium (Figure 3B) with occasional foci of adipose tissue and blood vessels could also be identified (Figure 4). Other mature or immature elements were not seen, even on extensive sampling. On immunohistochemistry (IHC), tumor cells were positive for synaptophysin and glial fibrillary acidic protein. A final diagnosis of primary retroperitoneal mature cystic teratoma with predominance of neurogenic elements was made.

The patient was discharged uneventfully in a stable condition. Post operative 1 year follow up failed to reveal any tumor recurrence.

\section{DISCUSSION}

Primary retroperitoneal neoplasms are a rare but diverse group of benign and malignant tumors that arise within the retroperitoneal space but outside the major organs in this space. They can be solid or cystic masses, each of which can be further subdivided into neoplastic and non-neoplastic masses. Of the primary retroperitoneal neoplasms, $70 \%-$ $80 \%$ are malignant in nature, and these account for $0.1 \%$ $0.2 \%$ of all malignancies in the body (4). Among them, primary retroperitoneal teratomas are extremely unusual neoplasms accounting for approximately $1-11 \%$ of all 

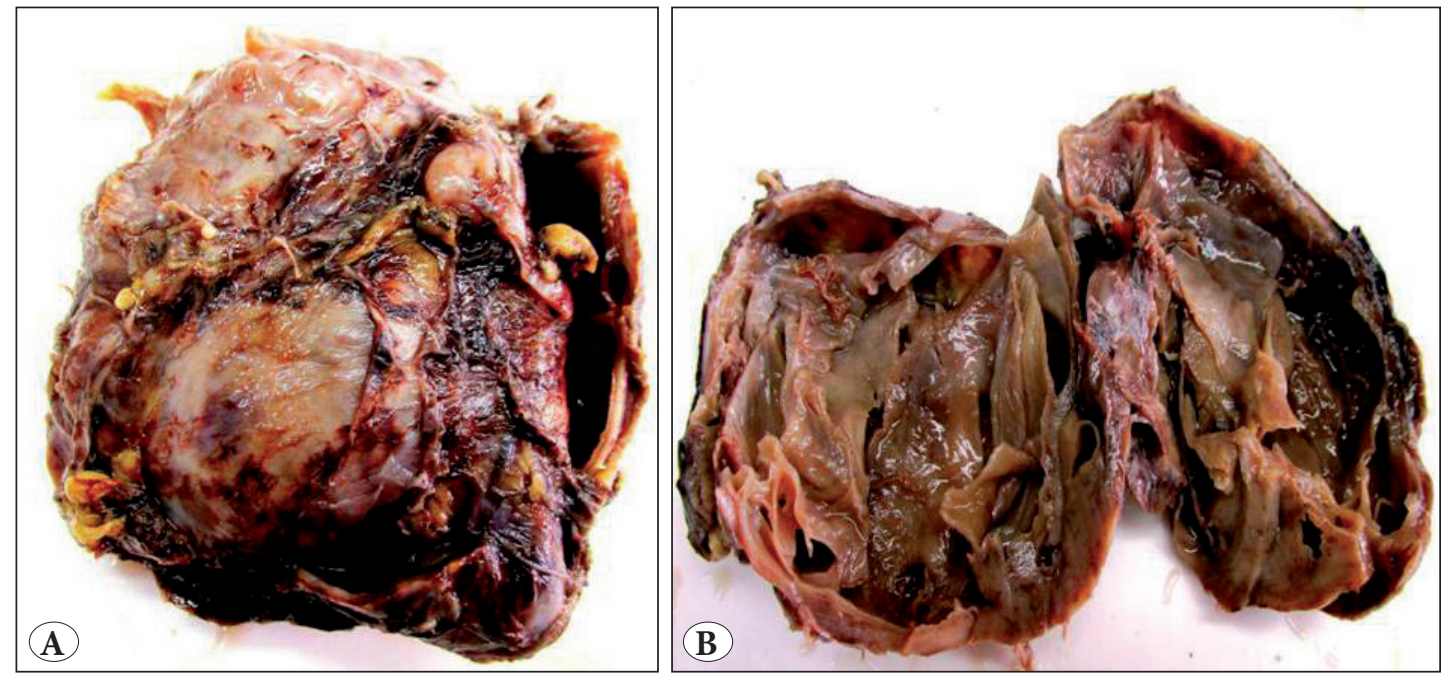

Figure 2:

A) Gross specimen of retroperitoneal mass. B) Cut section revealing multilocular cystic component of the tumor.
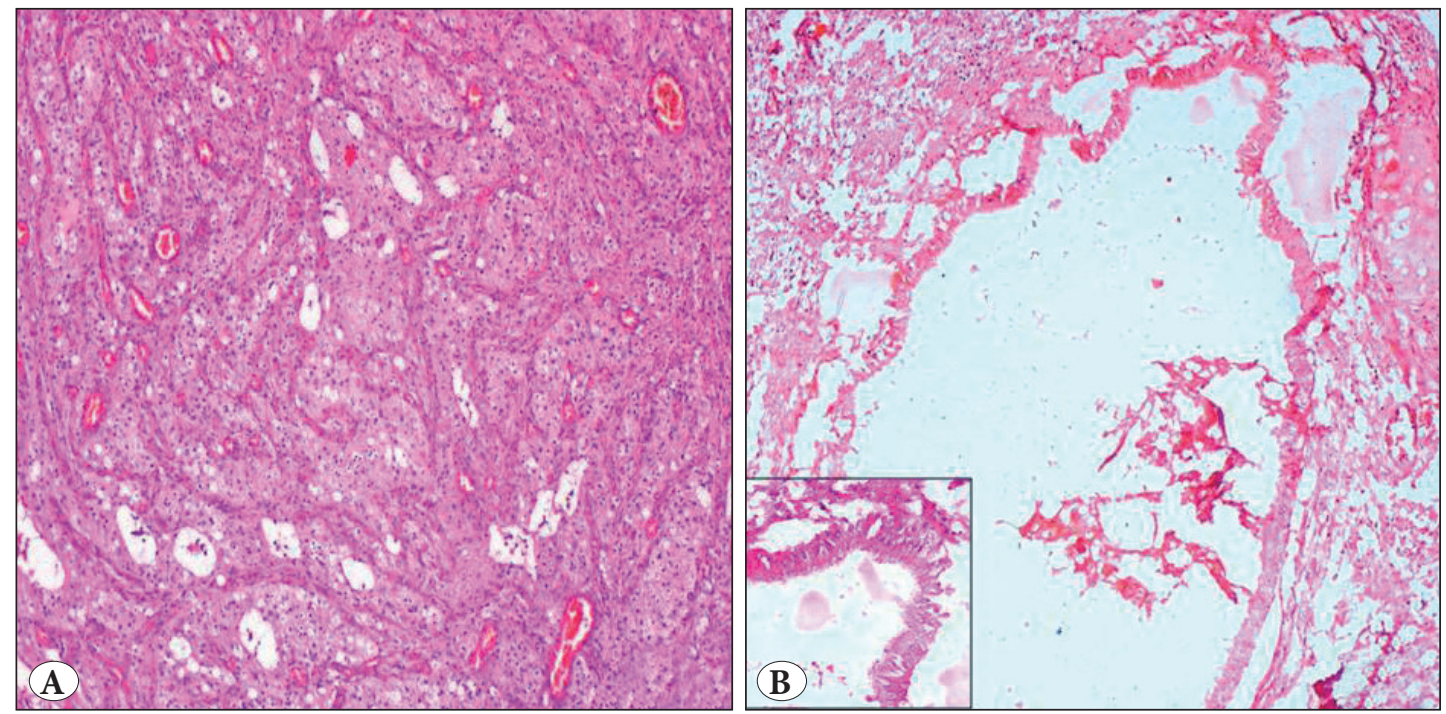

Figure 3:

A) Photomicrograph showing predominantly mature glial tissue (H\&E; x200). B) Glandular structure lined by ciliated columnar epithelium (H\&E; x200) [Inset: Ciliated columnar epithelium (H\&E; x400)]

primary retroperitoneal neoplasms and typically occurs in neonates, infants, and children (5).

Primary retroperitoneal teratomas involving adrenal glands are exceedingly uncommon accounting for only $4 \%$ of all primary teratomas (6) and can be mistaken for adrenal neoplasms (7). As in our case, based on radiology, the left adrenal gland was inseparable from the mass, giving an appearance that the tumor might have arisen from the adrenal gland. Such an unusual radiological finding may cause erroneous diagnosis. However, laboratory investigations including serum tumor markers ruled out the possibility of adrenal gland being the possible origin of this tumor.

The diagnosis of this tumor is based on a combination of high index of clinical suspicion, laboratory and radiological investigations, though histopathology is the gold standard.

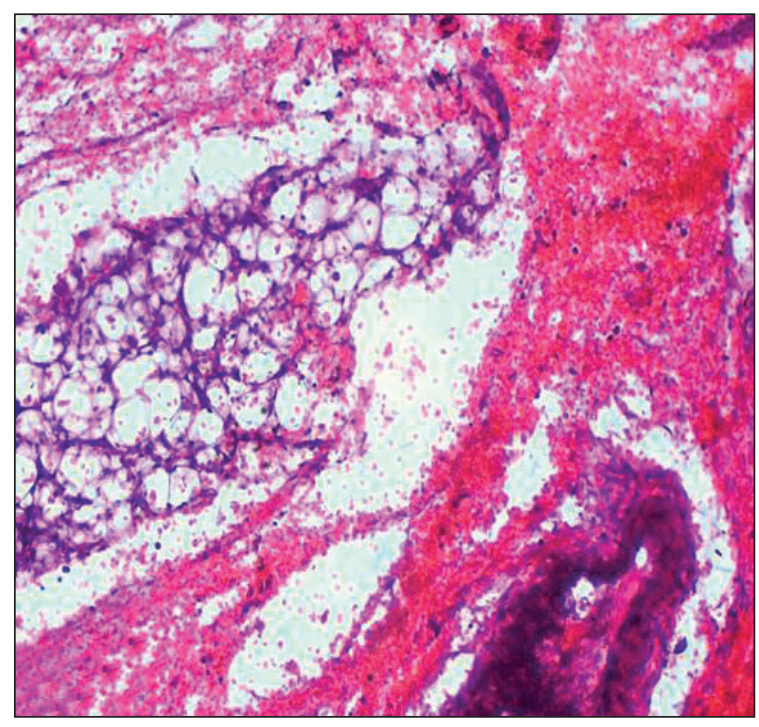

Figure 4: Adipose tissue and blood vessels (H\&E; x400). 
These tumors are usually asymptomatic but may manifest as abdominal/back/flank pain, abdominal distention, or a palpable abdominal mass, like in our case. Other symptoms can be of genito-urinary or gastrointestinal tract, limb/ genital swelling and secondary infections. Rarely malignant transformation and an acute syndrome can occur, involving peritonitis, intestinal obstruction, or renal colic (8). Laboratory investigations including serum tumor markers played a pivotal role in our patient, in clinching the diagnosis. The retroperitoneal teratomas have a property of expressing various serum tumor markers such as elevated AFP, CEA and CA 19-9. These markers can also be used to monitor successful treatment or detect relapse in patients with specific tumor marker-secreting teratomas as suggested by few authors (9).

Radiology has proved to be a valuable pre-operative diagnostic tool but has its own limitations $(10,11)$. Plain $\mathrm{X}$-ray can demonstrate calcified material while USG can differentiate between cystic and solid elements. CECT can help to determine the size, extent of the tumor, relationship to vessels and in differential diagnosis. Magnetic Resonance Imaging (MRI) can offer better assessment of tumor staging and distinction between benign and malignant neoplastic features (12). In our case, no calcification was seen both on USG or CECT, and MRI was not done, owing to the unaffordability by the patient.

Various differential diagnosis of retroperitoneal cystic lesions are cysts (mesenteric, omental, splenic, enteric duplications, mullerian, epidermoid, tailgut), solid neoplasms with cystic change (paraganglioma, neurilemmomas, leiomyosarcomas), lymphangiomas, lymphangioleiomyomas, mucinous/serous cystadenoma or cystadenocarcinoma, haematoma, urinoma, lymphocoele, pancreatic and nonpancreatic pseudocyst.

Complete surgical excision, either by open surgery or by laparoscopy followed by histopathology evaluation is the mainstay for its definitive diagnosis as well as treatment $(1,13)$. Usually teratomas, histopathologically consists of multiple parenchymal tissues that are derived from more than one germ cell layer (6). Our case was interesting as numerous sections were taken to locate the different elements of teratoma microscopically, but we could only find predominantly mature neurogenic element. However, after extensive sampling, a glandular structure and a tiny focus of adipose tissue could be identified. Therefore, this case cannot be considered as pure monodermal. Thus, we designated this case as primary mature cystic retroperitoneal teratoma with predominance of neurogenic elements. An extensive search of PubMed and Medline revealed one case with similar predominance of neurogenic elements but that was in an ovarian mature cystic teratoma (14). The pathogenesis of these predominant specific tissues in teratoma is still obscure. In our case, teratoma components were mature but approximately $90 \%$ of the tumor area showed glial tissue. Hence, the current case becomes a relevant value addition to the existing world literature.

The prognosis of primary retroperitoneal teratomas is generally good if the tumor is removed completely (15). In our case, the tumor was totally excised and the postoperative as well as the follow up period was uneventful. Hence, it is postulated that these tumors with predominant neurogenic elements also behave in a benign manner as the primary retroperitoneal mature teratomas do. However, a close follow up is mandatory as recommended by few researchers because of the possibility of its malignant transformation $(12,16)$.

In conclusion, primary retroperitoneal teratoma, though a rare entity, should always be considered among differentials of adrenal masses, as it can masquerade a primary adrenal tumor, as seen in our case. Preoperatively laboratory and radiological investigations do play an integral role, but it is the histopathology which is confirmative. More insight is required to understand the genesis and behaviour of these tumors with one predominant element in teratomas.

\section{CONFLICT OF INTEREST}

The authors declare no conflict of interest.

\section{REFERENCES}

1. Mathur P, Lopez-Viego MA, Howell M. Giant primary retroperitoneal teratoma in an adult: A case report. Case Rep Med. 2010;2010. pii: 650424 .

2. Bedri S, Erfanian K, Schwaitzberg S, Tischler AS. Mature cystic teratoma involving adrenal gland. Endocr Pathol. 2002;13:59-64.

3. Sharma S, Singh M, Bhuyan G, Mandal AK. Extragonadal dysgerminoma presenting as neck metastasis and masquerading as a thyroid swelling. Clin Cancer Investig J. 2016;5:43-5.

4. Neville A, Herts BR. CT characteristics of primary retroperitoneal neoplasms. Crit Rev Comput Tomogr. 2004;45:247-70.

5. Schmoll H. Extragonadal germ cell tumors. Ann Oncol. 2002;13:265-72.

6. Polo JL, Villarejo PJ, Molina M, Yuste P, Menendez JM, Babe J, Puente S. Giant mature cystic teratoma of the adrenal region. AJR Am J Roentgenol. 2004;183:837-8.

7. Hui JP, Luk WH, Siu CW, Chan JC. Teratoma in the region of an adrenal gland in a 77-year-old man. J Hong Kong Coll Radiol. 2004;7:206-9.

8. Wolski Z, Jasinski Z. Retroperitoneum teratoma. Int Urol Nephrol. 1981;13:137-40. 
9. McKenney JK, Heerema-McKenney A, Rouse RV. Extragonadal germ cell tumors: A review with emphasis on pathologic features, clinical prognostic variables, and differential diagnostic considerations. Adv Anat Pathol. 2007;14:69-92.

10. Shinagare AB, Jagannathan JP, Ramaiya NH, Hall MN, Van den Abbeele AD. Adult extragonadal germ cell tumors. AJR Am J Roentgenol. 2010;195:W274-80.

11. Barka M, Mallat F, Hmida W, Ahmed KB, Chavey SO, Abdallah $\mathrm{AB}$, Tlili K. Giant primary retroperitoneal teratoma in an adult male: A rare entity. Int J Case Rep Images. 2014;5:558-61.

12. Chaudhary A, Misra S, Wakhlu A, Tandon RK, Wakhlu AK. Retroperitoneal teratomas in children. Indian J Pediatr. 2006;73:221-3.
13. Ratkala JM, Shaikb NJ, Salia D, Choukimatha SM. Rare primary retroperitoneal teratoma masquerading as adrenal incidentaloma. African Journal of Urology. 2015;21:96-9.

14. Akbulut M, Kelten EC, Ege CB. Mature cystic teratoma with predominately neurogenic elements-case report. Aegean Pathology Journal. 2006;3:18-20.

15. Aldhilan A, Alenezi K, Alamer A, Aldhilan S, Alghofaily K, Alotaibi M. Retroperitoneal teratoma in 4 months old girl: Radiology and pathology correlation. Curr Pediatr Res. 2013; 17:133-6.

16. Okulu E, Ener K, Aldemir M, Isik E, Irkkan C, Kayigil O. Primary mature cystic teratoma mimicking an adrenal mass in an adult male patient. Korean J Urol. 2014;55:148-51. 